\title{
Oral insulin improves metabolic parameters in high fat diet fed rats
}

\author{
LEANDRO C. LIPINSKI ${ }^{1}$, LOUISE B. KMETIUK ${ }^{2}$, PAULO C.F. MATHIAS ${ }^{3}$, ANANDA \\ MALTA $^{3}$, GIOVANI M. FAVERO ${ }^{2}$, TATIANE A. RIBEIRO ${ }^{3}$, ALCEU TOLEDO $^{1}$, \\ MARIO R. MONTEMOR NETTO ${ }^{1}$ and MARCOS R.S. RODRIGUES ${ }^{1}$ \\ ${ }^{1}$ Department of Medicine, State University of Ponta Grossa, Av. General Carlos \\ Cavalcanti, 4748, Bairro Uvaranas, 84030-900 Ponta Grossa, PR, Brazil \\ ${ }^{2}$ Department of Pharmaceutical Sciences, State University of Ponta Grossa, Av. General \\ Carlos Cavalcanti, 4748, Bairro Uvaranas, 84030-900 Ponta Grossa, PR, Brazil \\ ${ }^{3}$ Laboratory of Secretion Cell Biology, Department of Biotechnology, Genetics and Cell Biology, State \\ University of Maringá, Av. Colombo, 5790, Jd. Universitário, 87020-900 Maringá, PR, Brazil
}

Manuscript received on January 26, 2017; accepted for publication on April 29, 2017

\begin{abstract}
Introduction/Aim: The gut has shown to have a pivotal role on the pathophysiology of metabolic disease. Food stimulation of distal intestinal segments promotes enterohormones secretion influencing insulin metabolism. In diabetic rats, oral insulin has potential to change intestinal epithelium behavior. This macromolecule promotes positive effects on laboratorial metabolic parameters and decreases diabetic intestinal hypertrophy. This study aims to test if oral insulin can influence metabolic parameters and intestinal weight in obese non-diabetic rats. Methods: Twelve weeks old Wistar rats were divided in 3 groups: control (CTRL) standard chow group; high fat diet low carbohydrates group (HFD) and HFD plus daily oral 20U insulin gavage (HFD+INS). Weight and food consumption were weekly obtained. After eight weeks, fasting blood samples were collected for laboratorial analysis. After euthanasia gut samples were isolated. Results: Rat oral insulin treatment decreased body weight gain $(p<0,001)$, fasting glucose and triglycerides serum levels $(\mathrm{p}<0,05)$ an increased intestinal weight of distal ileum $(\mathrm{P}<0,05)$. Animal submitted to high fat diet presented higher levels of HOMA-IR although significant difference to CT was not achieved. HOMA-beta were significantly higher $(\mathrm{p}<0.05)$ in HFD+INS. Visceral fat was $10 \%$ lower in HFD+INS but the difference was not significant. Conclusions: In non-diabetic obese rats, oral insulin improves metabolic malfunction associated to rescue of beta-cell activity.
\end{abstract}

Key words: oral insulin, intestine, diabetes, obesity, metabolism, pancreatic beta-cell.

\section{INTRODUCTION}

For decades, oral insulin delivery has been studied in humans and different experimental models (Murlin et al. 1940, Florence 2015, Sheng et al.

Correspondence to: Marcos Ricardo da Silva Rodrigues

E-mail: marcosrsr75@gmail.com
2016). Although the peptide insulin is destroyed in acid environment of stomach, by not completely understood means it promotes serum metabolic parameters improvement as well as promotes function and morphological changes in gut epithelium (Sukhotnik et al. 2011, Shehadeh et al. 2006). 
The gastrointestinal tract has shown to have a pivotal role on metabolic disease development (Santoro 2008). Diabetes promotes an enhancement of intestinal weight and absorptive capacity, being observed that T2DM patients have more intestinal mass than euglycemic counterparts (Fujita et al. 1998, Noda et al. 2001, Verdam et al. 2011).

In rats, oral insulin is able to reverse diabetic intestinal hyperplasia (Sukhotnik et al. 2011). Differential intestinal insulin receptors expression under different insulin serum levels might be link between oral insulin and intestinal epithelium behavior.

Although results of oral insulin treatment in diabetic animals is well documented in literature, no study has showed results of this intervention in non diabetic obese animals promoted by high fat diet.

This study analyze the effect of oral insulin in non-diabetic obese animal fed with high fat low carbohydrate chow.

\section{MATERIALS AND METHODS}

Twelve weeks age Wistar rats (190 gr) were divided in 3 groups: A: standard chow Nuvilar ${ }^{\circledR}$ CTRL (10), B: (HFD) high fat diet (10) and C: (HFD+INS) high fat diet plus oral regular insulin (10) in daily doses of $20 \mathrm{U}$ administered by oral gavage. High fat diet was obtained by adding lard on regular chow and presented lower levels of carbohydrates. Standard diet composition (\% Kcal) were $68 \%$ of carbohydrates, $22,7 \%$ of protein and $9,3 \%$ of fat and $39,1 \%$ of $\mathrm{CH}, 16 \%$ of protein and $44,6 \%$ of fat on high fat diet. Food consumption was obtained by daily measurement of residual diet on five animal cages. Animal were weighed weekly.

After 8 weeks of experiment, twelve hour fasted animal were anesthetized with intraperitoneal xylazin $(5 \mathrm{mg} / \mathrm{kg})$ and ketamine $(40 \mathrm{mg} / \mathrm{kg})$. Blood samples were obtained by internal jugular vein puncture and centrifugated. Glucose, triglycerides, total cholesterol and insulin serum levels were measured. Animals were euthanized.

Visceral fat (retroperitoneal and epididymal) were resected. The small intestine was removed, stripped of adherent fat and mesentery, washed with normal saline and dried. The removed bowel was divided into three segments: duodenum (10 $\mathrm{cm}$ from pylorus to ligament of Treitz ), proximal jejunum (10 cm from the ligament of Treitz) and distal ileum $(10 \mathrm{~cm}$ proximal to the ileo-cecal junction). Each segment was weighed. Bowel weights were calculated as $\mathrm{mg} / 10 \mathrm{~cm}$ bowel length/100 of body weight.

\section{RADIOIMMUNOASSAY AND BIOCHEMICAL ANALYSES}

Plasma insulin was measured by radioimmunoassay (RIA), in gamma couter (Wizard2 Automatic Gamma Counter, TM-2470, PerkinElmer ${ }^{\circledR}$, Shelton, CT, USA). It was used as standard the human insulin; the antibody used was the anti-rabit insulin antibody (Sigma-Aldrich ${ }^{\circledR}$, St. Louis, MO, USA) and radioactive insulin used was the labeled Iodo125 recombinant human insulin (PerkinElmer, Shelton CT, USA). The intra-assay coefficients of variation were in the range $8-10 \%$. The limit of detection was $0.006 \mathrm{ng} / \mathrm{ml}$.

The measurements were performed in a single assay. Glucose and lipid profiles were measured with a commercial kit Roche $^{\circledR}$ Indianapolis, IN, USA). The homeostasis model assessment of insulin resistance (HOMA-IR) was used as the physiological index of insulin resistance. Homeostasis model of beta cell function HOMABETA was used to predict secretory capacity of pancreatic beta cells. This was assessed from fasting glucose and fasting insulin concentrations using the following formula: HOMA-IR = (fasting insulin $[\mathrm{ng} / \mathrm{mL}] \times$ fasting glucose $[\mathrm{mg} / \mathrm{dL}]) / 22.5$ and (fasting insulin $[\mathrm{ng} / \mathrm{mL}] \times 20 /$ fasting glucose $[\mathrm{mg} / \mathrm{dL}])-3,5)$ respective. 


\section{STATISTICAL ANALYSIS}

Data were presented as mean \pm standard deviation (SD). Afterward, the data were subjected to ANOVA (one-way) and Tukey's post hoc test or Student $t$ test when necessary. $\mathrm{P}<0.05$ was considered significantly different. Tests and graphics were performed using GraphPad Prism version 6.01 for Windows (GraphPad Software, Inc. San Diego, CA, USA).

\section{RESULTS}

ORAL INSULIN REDUCES BODY WEIGHT (BW) IN HIGH FAT DIET ANIMALS

Figure 1 shows the bw evolution analyzed by area under the curve (AUC) analysis HFD+INS $(43620 \pm 4257)$ group was $10,6 \%$ lower than HFD $(48813 \pm 3735)$ rats $(p<0.001)$ (Figure 1a). There was no difference of food consumption between HFD and HFD+INS groups. Animals treated with HFD showed lower food consumption than control animals, regardless of the insulin treatment (Figure 1b). HFD animals presented significant increase of visceral fat pad stores $(20,87 \pm 7,39)$ than CTRL $(14,02 \pm 3,51)(\mathrm{p}<0,05)$. The difference was not observed between Control and HFD+INS $(18,73 \pm 6,86)$ (ANOVA analysis) Oral insulin administration promoted a reduction of $10 \%$ in visceral fat pad stores compared to HFD group, however the difference did not reach significance.

ORAL INSULIN REDUCES SERUM GLUCOSE LEVELS AND ENHANCES HOMA BETA INDEX IN HIGH FAT DIET ANIMALS

ANOVA analysis showed significant difference of fasting glucose levels $(p<0,05)$ between HFD $(170,1 \pm 20,33)$ and CTRL $(145,2 \pm 8,63)$ and between HFD and HFD+INS $(155,8 \pm 13,49)(\mathrm{p}<0,05)$ (Figure 2b). HFD group had lower level of HOMA BETA index than CTRL (not significant). Oral insulin (HFD+INS) promoted a significant increase of HOMA-BETA index $(43,72 \pm 27,98)$ compared to $\operatorname{HFD}(24,3 \pm 19,25)(p<0,05)$ (Figure $2 b)$.

$\operatorname{HFD}(0,094 \pm 0,07)$ and $\operatorname{HFD}+\operatorname{INS}(0,093 \pm 0,05)$ animals showed higher levels of HOMA-IR compared to CTRL $(0,054 \pm 0,02)$ (Figure $2 \mathrm{c}$ ) although differences among groups were not significant. Compared to CTRL, fasting insulin was $60 \%$ higher in HFD and $30 \%$ in HFD+INS
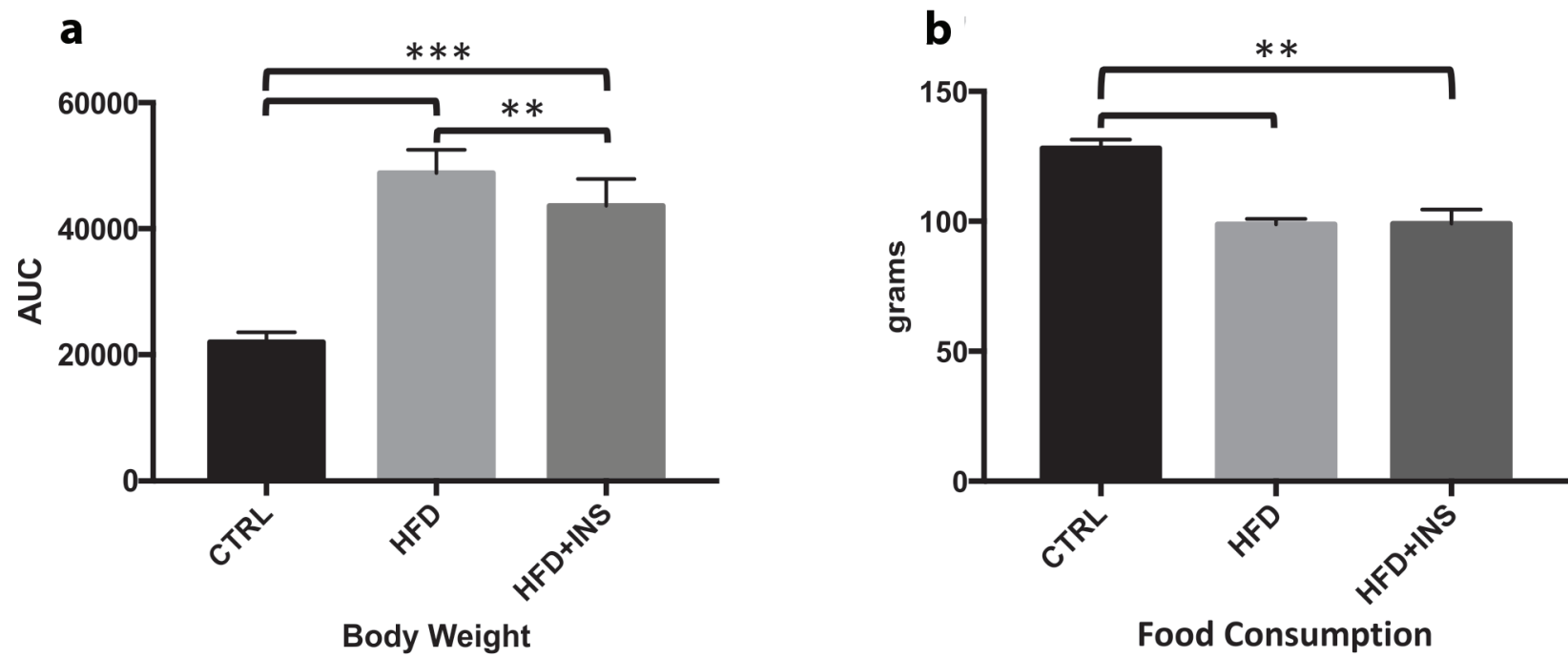

Figure 1 - (a) - Body weight changes in CTRL animal (standard chow) $(\mathrm{n}=10)$ and in HFD (rich in fat e low carbohydrate chow) $(\mathrm{n}=10)$ and HFD+INS (plus oral insulin gavage) $(\mathrm{n}=10)$. Area Under Curve (AUC). $* \mathrm{p}<0,05$ HFD versus HFD+INS, $<<0,01 \mathrm{CTRL}$ versus HFD and CTRL versus HFD+INS. (b) - Food Consumption (grams/week). ${ }^{* *} \mathrm{p}<0,01$ CTRL versus HFD and HFD+INS. 

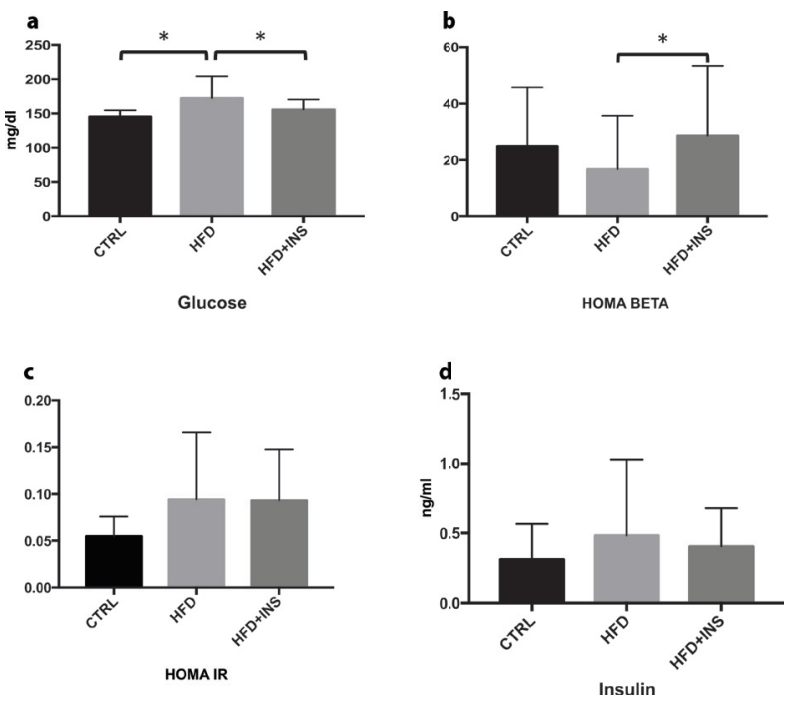

Figure 2 - (a) Fasting glucose, CTRL (Control group-regular chow), HFD (rich in fat and low carbohydrates chow) and HFD + INS (plus oral insulin gavage). Values are mean \pm SD. ${ }^{*} \mathrm{p}<0,05$ CTRL versus HFD and HFD versus HFD+INS. (b) HOMA BETA index, ${ }^{*} \mathrm{p}<0,05$ HFD versus HFD+INS. (c) HOMA IR index. (d) Insulin fasting levels.

however we did not observe significant difference. (Figure 2d).

\section{ORAL INSULIN REDUCES TRIGLYCERIDES \\ LEVELS IN HIGH FAT DIET FED ANIMALS}

Control group presented higher levels of triglycerides serum levels $(114,7 \pm 30,47)$ than HFD $(103,6 \pm 27,7)$ and HFD+INS $(85,17 \pm 24,77)$ group. The difference was significant between CTRL and HFD+INS (ANOVA p <0,05) (Figure 3). When analyzed by $T$ test, comparing animals with same diet, oral insulin significantly reduced serum triglycerides $(\mathrm{P}<0,05)$. Cholesterol levels were not different between groups.

\section{ORAL INSULIN ENHANCES SEGMENTAL WEIGHT OF DISTAL ILEUM}

Control animals fed with standard diet presented higher intestinal mass (segment weight $\mathrm{mg} / 10 \mathrm{~cm} / 100 \mathrm{~g}$ body weight) in all segments analyzed (duodenum, jejunum and ileum) compared to animals fed with high fat low carbohydrates diet

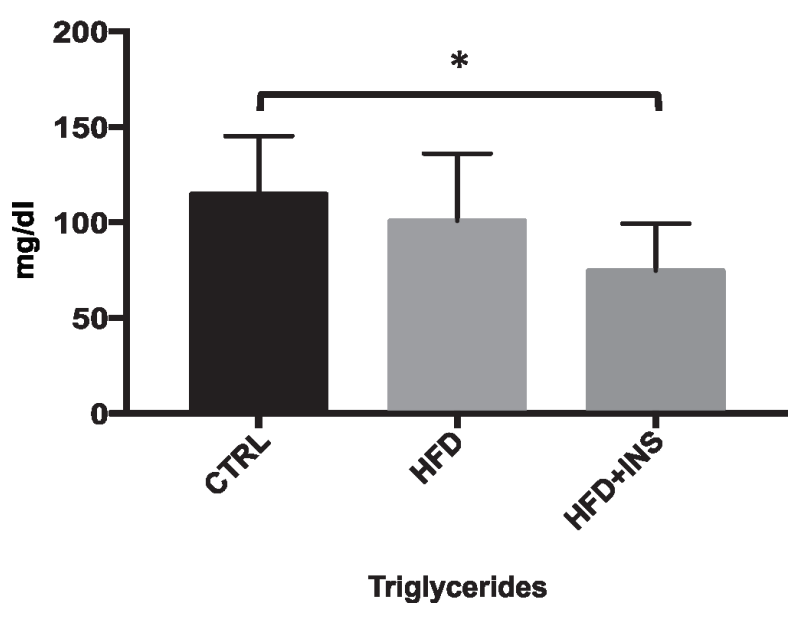

Figure 3 - Fasting triglycerides. CTRL (Control group-regular chow), HFD (rich in fat and low carbohydrates) and HFD+INS (oral insulin gavage). Values are mean $\pm \mathrm{SD}$. ${ }^{*} \mathrm{p}<0,05 \mathrm{CTRL}$ versus HFD+INS.

$(\mathrm{p}<0,001)$ (Figure 4a). Comparing groups with the same diet (HFD and HFD+INS) intestinal proportion was $0.2039 \pm 0.04$ (duodenum), $0.15 \pm 0.0239$ (jejunum), $0.147 \pm 0.016$ (ileum) in HFD and $0.218 \pm 0.032$ (duodenum), $0.1816 \pm 0.036$ (jejunum), $0.235 \pm 0.09$ (ileum) in HFD + INS. Oral insulin did not influence weight of duodenum, promoted a trend of increasing weight of jejunum and significantly enhanced intestinal mass of distal ileum portion of intestine $(p<0,05)$ (Figure $4 b)$.

\section{DISCUSSION}

For decades, scientists have looked for a more physiological route to deliver insulin (Florence 2015). The fact that insulin like other polypeptides is destroyed in acid environment of the stomach and by pancreatic enzymes turned this option unattractive (Sonia and Sharma 2014). Although not absorbed in its biologically active form, oral administered insulin induces metabolic, antrophometric and gut morphology alterations (Sukhotnik et al. 2011).

Oral administered insulin promotes different outcome in intestinal epithelium under different glucose and insulin serum levels. In diabetes, 

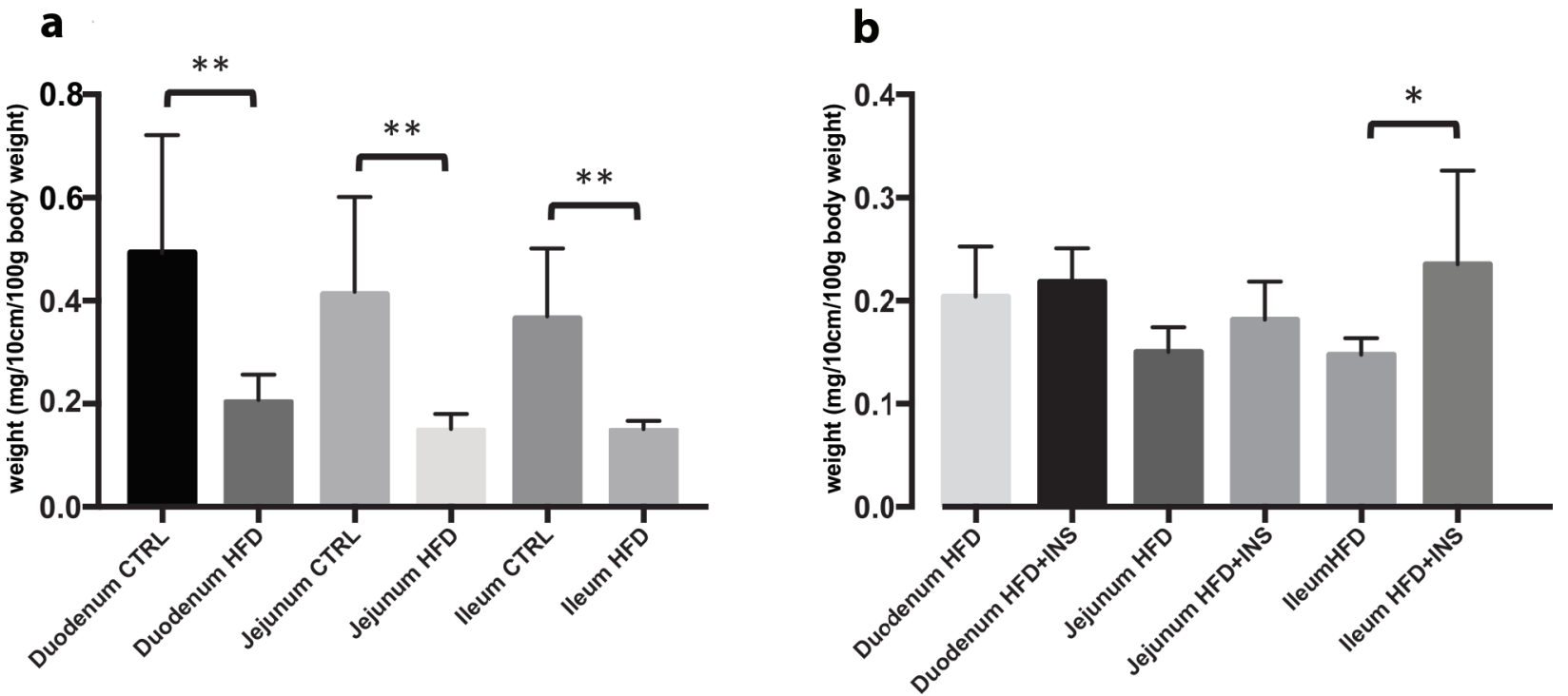

Figure 4 - a. Effect of diet in intestinal epithelium segments $(10 \mathrm{~cm})$ weight (duodenum, jejunum and ileum). CTRL (regular chow), HFD (high fat low carbohydrates diet) $(\mathrm{n}=10)$. Values are mean \pm SD. $* * \mathrm{p}<0,001$. b. Effect of insulin in intestinal segments $(10 \mathrm{~cm})$ weight (duodenum, jejunum and ileum). HFD (high fat low carbohydrates diet) (n=10), HFD+INS (high fat low carbohydrates diet plus oral insulin gavage) $(\mathrm{n}=10)$. Values are mean \pm SD. ${ }^{*} \mathrm{p}<0,05$.

an hypertrophic phenomenon is observed in intestinal epithelium leading to hyperplasia and an enhancement of absorptive capacity (Fujita et al. 1998). Oral insulin reverses this "hypertrophy" returning the epithelium to its normal aspect. Verdam et al. (2011) proposed that obese diabetic patients have a higher intestinal mass than non diabetic obese, being suggested (Rodrigues et al. 2015, Fujita et al. 1998) that intestinal alteration might be a cornerstone factor for diabetes evolution.

To date, no study has shown intestinal alteration resulting from oral insulin administration to obese animals. In this experimental model, animals were offered a high fat and low carbohydrate chow. Interestingly these animals presented a strong decreasing of intestinal weight in all analyzed segments. Low concentration of carbohydrates (CH) might be the answer for this outcome. $\mathrm{CH}$ are essential for intestinal mucosal growth. Animal under parenteral nutrition has less intestinal mass than its counterparts. When luminal $\mathrm{CH}$ are restored, significant mucosal recovery is achieved. The intestinal epithelium growth is proportional to diet glucose load levels (Weser et al. 1982). In this study oral insulin promoted an enhancement of intestinal weight that was only significant in distal ileum. There was no alteration in duodenum and a trend of higher growth in jejunum (Figure 4a).

In rats subjected to massive intestinal resection, oral insulin administration on post surgery period promoted enhancement of intestinal mass mainly in distal segments of ileum (Sukhotnik et al. 2005), a similar pattern of intestinal morphologic alterations observed in our experiment. Interestingly this outcome regarding intestinal epithelium growth under oral insulin administration is opposed to the observed in diabetic rats, where a reduction of intestinal mass by oral insulin is observed (Sukhotnik et al. 2011).

Actually, distal ileum seems to be a cornerstone segment in metabolic diseases development. Bariatric surgery procedures dramatically improve metabolic parameters even before losing weight. Exclusion of proximal intestine from food transit (foregut hypothesis) and distal intestinal segments stimulation by food (hindgut hypothesis) have 
been pointed as main factors for metabolic parameters amelioration after surgery (Rubino et al. 2006). Actually, distal ileum presents a higher concentration of incretin secretion entero-endocrine cells such as L cells, that, among other hormones, are responsible for glucagon peptide-1 (GLP-1) secretion. Higher intestinal mass enhance the pool of incretin secreting cells, potentiating its secretion (Mumphrey et al. 2013). An interesting capacity of GLP-1 is to enhance energy expenditure (Tomas et al. 2015, Goldsmith et al. 2015).

High fat diet animal presented lower food consumption than control group, probably owing to higher caloric content of pellets inducing higher satiety. Although same food consumption values in groups HFD and HFD+INS, weight gain was significantly lower in the insulin treated group. Other studies showed similar results in euglycemic rats. Ribeiro et al. (unpublished data) also observed lower weight gain and metabolic improvement in rat litters fed with higher insulin levels in mother milk.

A very intriguing result of this study is the difference in glucose levels between groups. Although reduction of serum glucose levels with oral insulin treatment was observed in other studies, we first identified a significantly higher HOMA beta index in oral insulin treated animal leading us to infer a rescue of beta cell function in insulin group. Better beta cell function might be also the answer for lower triglycerides levels in intervention group. Interestingly, this study showed a trend of reduction $(p<0,1)$ of TG levels in high fat diet treated animals. Some authors have shown benefits of high fat diet with low $\mathrm{CH}$ diets (Hu et al. 2012) to decrease metabolic risk factors. The diet offered in this study fulfills this criteria.

The metabolic improvement in this study was paralleled to a higher distal intestinal mass promoted by oral insulin administration and this might be the answer for different metabolic parameters improvement encountered. Ongoing studies focusing on postprandial metabolic parameters will probably provide us this information.

\section{CONCLUSIONS}

In non-diabetic obese rats, oral insulin treatment enhance distal intestinal mass and improves metabolic malfunction associated to beta-cell activity rescue.

\section{REFERENCES}

FLORENCE AT. 2015. Oral insulin delivery: a chimera? Int J Pharm 495(1): 218-219.

FUJITA Y, KOJIMA H, HIDAKA H, FUJIMIYA M, KASHIWAGI A AND KIKKAWQ R. 1998. Increased intestinal glucose absorption and postprandial hyperglycemia at the early step of glucose intolerance in Otsuka Long- Evans Tokushima fatty rats. Diabetologia 41: 1459-1466.

GOLDSMITH F, KEENAN MJ, RAGGIO AM, YE X, HAO Z, DURHAM H, GEAGHAN J, JIA W, MARTIN RJ AND YE J. 2015 Induction of Energy Expenditure by Sitagliptin Is Dependent on GLP-1 Receptor. PLoS One 10(5): e0126177.

HU T, YAO L, REYNOLDS K, WHELTON PK, NIU T, LI S, HE J AND BAZZANO LA 2012. Effects of LowCarbohydrate Diets Versus Low-Fat Diets on Metabolic Risk Factors: A Meta-Analysis of Randomized Controlled Clinical Trials. Am J Epidemiol 176(Suppl 7): S44-S54.

MUMPHREY MB, PATTERSON LM, ZHENG H AND BERTHOUD HR. 2013. Roux-en-Y gastric bypass surgery increases number but not density of CCK-, GLP-1-, 5-HT-, and neurotensin-expressing enteroendocrine cells in rats. Neurogastroenterol Motil 25: e70-e79.

MURLIN JR, GIBBS CB, ROMANSKY MJ, STEINHAUSEN TB AND TRUAX FL. 1940. Effectiveness of per-oral insulin in human diabetes. Only mildly encouraging results. J Clin Invest 19(5): 709-722.

NODA T, IWAKIRI R, FUJIMOTO K, YOSHIDA T and UTSUMI H. 2001. Suppression of Apoptosis Is Responsible for Increased Thickness of Intestinal Mucosa in Streptozotocin-Induced Diabetic Rats. Metabolism 50(3): 259-264.

RODRIGUES MR, SANTO MA, FAVERO GM, VIEIRA EC, ARTONI RF, NOGAROTO V, DE MOURA EG, LISBOA P AND MILLEO FQ. 2015. Metabolic surgery and intestinal gene expression: Digestive tract and diabetes evolution considerations. World J Gastroenterol 21(22): 6990-6998.

RUBINO F, FORGIONE A AND CUMMINGS DE. 2006. The mechanism of diabetes control after gastrointestinal bypass 
surgery reveals a role of the proximal small Intestine in the pathophysiology of type 2 diabetes. Ann Surg 244(5): 741-749.

SANTORO S. 2008. Is the Metabolic syndrome a disease of the foregut? Yes, excessive foregut. Ann Surg 247: 10741075.

SHENG J, HE H, HAN L, QIN J, CHEN S, RU G, LI R, YANG P, WANG J AND YANG VC. 2016. Enhancing insulin oral absorption by using mucoadhesive nanoparticles loaded with LMWP-linked insulin conjugates. J Control Release 233: $181-190$

SHEHADEH N, SUKHOTNIK I AND SHAMIR R. 2006. Gastrointestinal tract as a target organ for orally administered insulin. J Pediatr Gastroenterol Nutr 43(3): 276-281.

SONIA TA AND SHARMA CP. 2014. Oral Delivery of Insulin. Woodhead Publishing, Amsterdam.

SUKHOTNIK I, SHAMIR R, BASHENKO Y, MOGILNER JG, CHEMODANOV E, SHAOUL R, CORAN AG AND
SHEHADEH N. 2011. Effect of oral insulin on diabetesinduced intestinal mucosal growth in rats. Dig Dis Sci 56(9): 2566-2574.

SUKHOTNIK I, SHEHADEH N AND SHAMIR R. 2005. Oral insulin enhances intestinal re-growth following massive small bowel resection in rat. Dig Dis Sci 50: 2379Y 85.15.

TOMAS E, STANOJEVIC V, MCMANUS K, KHATRI A, EVERILL P, BACHOVCHIN WW AND HABENER JF 2015. GLP-1(32-36)amide Pentapeptide Increases Basal Energy Expenditure and Inhibits Weight Gain in Obese Mice. Diabetes 64(7): 2409-2419.

VERDAM FJ, GREVE JW, ROOSTA S, VAN EIJK H, BOUVY N, BUURMAN WA, AND RENSEN SS. 2011. Small Intestinal Alterations in Severely Obese Hyperglycemic Subjects. J Clin Endocrinol Metab 96(2): E379-E383.

WESER E, BABBITT J AND VANDEVENTER A. 1985. Relantionship between enteral glucose load and adaptative mucosal growth in the small bowel. Dig Dis Sci 30(7): 675-681. 